\title{
A laser Doppler system for monitoring of cerebral microcirculation: implementation and evaluation during neurosurgery
}

\author{
Peter Rejmstad, Gustav Åkesson, Oscar Åneman and Karin Wårdell
}

\section{Linköping University Post Print}

\section{Tweet}

N.B.: When citing this work, cite the original article.

The original publication is available at www.springerlink.com:

Peter Rejmstad, Gustav Åkesson, Oscar Åneman and Karin Wårdell, A laser Doppler system for monitoring of cerebral microcirculation: implementation and evaluation during neurosurgery, 2015, Medical and Biological Engineering and Computing.

http://dx.doi.org/10.1007/s11517-015-1332-5

Copyright: Springer Verlag (Germany)

http://www.springerlink.com/?MUD=MP

Postprint available at: Linköping University Electronic Press

http://urn.kb.se/resolve?urn=urn:nbn:se:liu:diva-120632 


\title{
A laser Doppler system for monitoring of cerebral microcirculation: implementation and evaluation during neurosurgery
}

\author{
Peter Rejmstad $^{1}$, Gustav Åkesson ${ }^{2,3}$, Oscar Åneman ${ }^{2,3}$, Karin Wårdell ${ }^{1}$ \\ ${ }^{1}$ Department of Biomedical Engineering, Linköping University, Sweden \\ ${ }^{2}$ Department of Neurosurgery, County Council of Östergötland, Sweden \\ ${ }^{3}$ Department of Clinical and Experimental Medicine, Linköping University, Sweden
}

Corresponding author:

Peter Rejmstad

Department of Biomedical Engineering

Linköping University

58185 Linköping

Sweden

Email: peter.rejmstad@liu.se

Telephone: +46101037704

Fax: +4613101902

Total number of words: 5373

Number of words of the abstract: 193

Number of Figures: 7

Number of Tables: 1 


\begin{abstract}
The aim of this study was to adapt and evaluate laser Doppler perfusion monitoring (LDPM) together with custom designed brain probes and software for continuous recording of cerebral microcirculation in patients undergoing neurosurgery. The LDPM system was used to record perfusion and backscattered light (TLI). These parameters were displayed together with the extracted heart rate (HR), pulsatility index (PI) and signal trends from adjustable time intervals. Technical evaluation was done on skin during thermal provocation. Clinical measurements were performed on ten patients undergoing brain tumour surgery. Data from 76 tissue sites were captured with a length varying between $10 \mathrm{~s}$ to $15 \mathrm{~min}$. Statistical comparisons were done using Mann-Whitney tests. Grey and tumour tissue could be separated from white matter using the TLI-signal $(\mathrm{p}<0.05)$. The perfusion was significantly higher in grey and tumour tissue compared to white matter $(\mathrm{p}<0.005)$. LDPM was successfully used as an intraoperative tool for monitoring local blood flow and additional parameters linked to cerebral microcirculation (perfusion, TLI, heart rate and PI) during tumour resection. The systems stability opens up for studies in the postoperative care of patients with e.g. traumatic brain injury or subarachnoid haemorrhage.
\end{abstract}

Keywords: Microcirculation, Brain Tumor, Laser Doppler Perfusion Monitoring (LDPM), Pulsatility Index (PI), Neurosurgery 


\section{Introduction}

Cerebral blood flow $(\mathrm{CBF})$ is a parameter of utmost importance in neurosurgery and intensive care for patients with a wide range of disorders including traumatic brain injury [29], subarachnoid haemorrhage and brain tumour. Imaging techniques such as positron emission tomography [22] and Xe-CT [14] for assessment of CBF provide only "snap-shot" information that in addition would require transportation of unstable, critically ill patients. Reliable bedside monitoring of regional CBF are not yet available in daily routine however recently this problem is being addressed as a part of multimodal brain monitoring $[3,2,1]$. Continuous postoperative monitoring of local $\mathrm{CBF}$ in the damaged brain could improve early detection of secondary insults and reduction in blood flow while assessing the need for medical intervention. A measurement system for continuous assessment of CBF-parameters during surgery and postoperative care need to rely on robust and stable methods to be able to make its way into clinical routine.

During the last decade the interest for optical brain monitoring has increased. However, a majority of the scientific investigations and optical systems are still in the animal studies phase in neuroscience $[13,39]$. Few of the optical techniques have so far made their way into daily routine in the neurosurgical operating theatre and neurointensive care. Examples of emerging optical blood flow techniques are laser speckle contrast imaging [25] and indocyanine green dilution [20]. Other examples are the use of laser Doppler perfusion monitoring (LDPM) [36,38] and imaging (LDPI) $[26,33]$. An advantage of probe based point measurements compared to imaging systems is that the probe can be directly inserted into brain tissue and look ahead into obstructed areas without the removal of healthy tissue. LDPM has so far been used for intraoperative navigation and assessment of cerebral microcirculation during deep brain stimulation implantations (DBS). In this setting, a long stiff probe with optical fibre designed to fit the stereotactic frame system was used for LDPM recordings along pre-calculated trajectories $[36,38]$. These studies show that LDPM is capable of monitoring the dynamics of the cerebral microvascular blood flow. Already twenty years ago, LDPM was suggested as CBF-monitoring device together with intracranial pressure measurements [11]. Recently Dreier et al. applied LDPM on the surface of the brain using an optode strip combined with electrodes to study spreading ischemia 2009 [7] and epileptic activity 2012 [35] in patients with subarachnoid haemorrhage. LDPM is, however, still not an established monitoring method in the neurosurgical and neurointensive care setting. In the present study a LDPM system with custom made brain probes and software is presented and evaluated in two steps. The two step evaluation was done firstly by experimental evaluation on skin, and secondly by recordings of perfusion and total backscattered light in ten patients during tumour surgery. 
Different approaches for a quick and easy overview of the dynamic processes in the acquired CBF-signals have been sought for [6]. The search for straightforward signal interpretations have resulted in various indexes where signal parameters are condensed to gain qualitative information [40]. From the perfusion signal it is easy to estimate heart rate (HR) as well as the so called pulsatility index (PI), commonly used for tracking blood flow changes which has been applied in transcranial Doppler and blood pressure measurements [5,4,10,31]. Furthermore the captured data need to be presented with a user friendly approach in order to optimize the feedback to the clinical interpreter. In this study we present an approach that support long-term recording of CBF-parameters by means of optics. The aim of the study was to introduce and evaluate LDPM together with custom designed probes and software for continuous recording of cerebral microcirculation in patients undergoing neurosurgery.

\section{Methods}

\subsection{Laser Doppler system, brain probe and software}

\subsubsection{Laser Doppler perfusion monitoring}

A LDPM device (Periflux 5000, Perimed AB, Sweden) was used in the system set up. It was connected to a personal computer with a data acquisition card (DAQ Card 6024E, National Instruments) and software (LabVIEW ${ }^{\mathrm{TM}}$ 2010, National Instruments Inc., USA). The custom made optical brain probes were used together with the LDPM device (Fig. 1a). Two signals are extracted from the system: the total backscattered light (TLI) and the perfusion (microvascular blood flow). The TLI signal is the summation of both AC and DC components from the photodetector and the perfusion signal represents the concentration of moving blood cells and their velocity. For details regarding signal processing see Nilsson et al. [24,23]. During intracerebral measurements, the TLI signal represents the diffuse reflection of light which is related to the tissue type whereas the perfusion is related to relative changes in the tissue's microcirculation. [36,38]. The total range of the perfusion and TLI signals are presented as 0 to 999 arbitrary units (a.u.) and 0 to 10.0 a.u. respectively. For all measurements, the DAQ sampling frequency $\left(\mathrm{f}_{\mathrm{s}}\right)$ was set to $100 \mathrm{~Hz}$. The probe was tested in Motility Standard solution (Perimed AB, Sweden) before each measurement occasion in order to verify comparable perfusion and TLI levels between measurements. If necessary the system was recalibrated.

\subsubsection{Brain probe}

The probe was designed to fit the neurosurgical application with a flexible tip for easy fixation and smooth insertion to the area of study. Four optical fibres $(\varnothing$ core $=125 \mu \mathrm{m}$, numerical aperture $=0.37$ ) were aligned along the interior of the probe and fixated next to each other at the tip (Fig. 1c). This 
corresponded to a fibre-fibre centre distance of $250 \mu \mathrm{m}$. The end of the probe tip was slightly rounded in order to avoid tissue trauma and could be fastened with sutures for stabilization during longer measurements. The probe's catheter-like design is similar to that of an external ventricular drain which enable continuous postoperative monitoring through a small burr hole in the skull and allows for probe extraction without reoperation. Two of the four optical fibres were connected to the LDPM device and the remaining two were available for complementary diffuse reflectance measurements- During the course of the project the probe design was revised and a second probe model was made, by changing the size of the exterior tip from $\varnothing=2 \mathrm{~mm}$ to $\varnothing=1.7 \mathrm{~mm}$ henceforth referred to as probe Model 1 and Model 2 respectively. The total length of the probe cable was approximately $5 \mathrm{~m}$ in order to keep the main equipment outside the sterile zone in the operating theatre. Prior to measurement the probe was sterilized using the STERRAD ${ }^{\circledR}$ protocol [15]. For further information regarding probe design see Rejmstad et al. [27].

\subsubsection{Software}

The software, developed in LabVIEW ${ }^{\mathrm{TM}}$ (National Instruments Inc., USA) made it possible to sample, store and display derived parameters and indexes of the captured LDPM signals as seen in Fig. 1b. The PI parameter $[5,4,10]$ is calculated according to literature as in equation 1 where $\operatorname{Perf} f_{\max }$ and Perf $f_{\min }$ corresponds to an average of the local (systolic) peaks and (diastolic) valleys in the perfusion signal respectively over the predefined time interval (10 s or more) while Perfaverage is the signal average over the same interval (see Fig. 4).

$$
P I=\frac{\text { Perf }_{\text {max }}-\text { Perf }_{\text {min }}}{\text { Perf }_{\text {average }}}
$$

Prior to the HR estimation the perfusion signal is low-pass filtered (cut-off frequency $20 \mathrm{~Hz}$ ) to remove high frequency noise and supress detection at the dicrotic notch. The online heart rate estimator uses peak finding to sort out and count the number of heart beats per selected interval and convert that into HR. Features of the monitoring software contain presentation of the perfusion and the TLI signal, as well as the display of the HR and PI. In order to present trend curves, various time interval (e.g. 10, 20,30 and $60 \mathrm{~s}$ ) can be set to calculate the average values fore presentation. Figure $1 \mathrm{~b}$ presents an example of the interface during a measurement.

\subsection{Experimental evaluation of system and probe}

The probe's function, were tested by measurements in Motility Standard. In order for the LDPM system to produce a perfusion signal with minimal noise, enough backscattered light from the tissue 
(TLI > 0.5) was required. The system was thereafter calibrated for the selected type of probe. This procedure was performed before and after cleaning the probe and sterilization according to the STERRAD $^{\circledR}$ protocol [15]. Secondly the probes were used for measurements of skin blood flow. The two probes (Model 1 and 2) were positioned on the face of the palm, about one mm apart, on one volunteering healthy person. Recordings were done before during and after a temperature provocation using an infrared heat source.

\subsection{Evaluation during neurosurgery}

\subsubsection{Patients}

Ten patients ( 6 female and 4 male, age 38 to 77 with an average of 59) referred for tumor surgery by the Department of Neurosurgery, Linköping University Hospital, where included in the clinical part of the study (Table 1). The patients were selected among individuals scheduled for craniotomy to enable measurement with direct contact to brain tissue. The selection was limited to surgical procedures where the research group has had previous experience with optical measurements. The clinical measurements were approved by the local ethics committee (M182-04, 2010/359-32) and written informed consent was received from the patient prior to each measurement.

\subsubsection{Surgical measurement procedure}

In order to evaluate the performances of the system and the brain probes in the clinical environment, measurements were done in relation to tumor resection and open skull biopsies. After opening of the skull the probe was manually inserted into a preselected brain tissue region while all other instruments and handheld devices were retracted to reduce the influence of movements that otherwise would affect the recorded signals. The probe was placed in three main types of tissue as defined by the surgeons' naked eye namely; white matter, grey matter and tumor. To receive stable signals the probe was kept at the same tissue site for at least $10 \mathrm{~s}$ up to several minutes, where the longest recording lasted for 15 min. In total 76 recordings were done at different tissue sites. Figure 2a presents an overview of the operation theatre and $2 \mathrm{~b}$ a close-up of the probe fixation in the brain tissue.

\subsubsection{Data analysis}

Recorded signals were sorted into grey matter, white matter and tumor tissue. The sorting was based on the surgeon's visual inspection and all statistical tests were performed using this type of classification. The data was tested for normal distribution by performing a Ryan-Joiner test using Minitab statistical software (Minitab Inc., USA) on the LDPM signals perfusion and TLI. The result from the test indicated that the data was not normally distributed. As a result medians of perfusion and TLI were used for each group. Mann-Whitney tests were used to investigate the difference between 
tissue groups. P-values $<0.05$ were considered significant. Tumor type was defined by routine histopathological examination and the tumours were graded as high (grade III-IV) or low (grade I-II) according to the WHO classification of tumours [21].

\section{Results}

\subsection{Experimental evaluation}

Before the skin measurements the probes where placed in motility standard solution to compare the measured values between probes. The calibration of the system involves adjustment of the perfusion level using Motility Standard. Measurements with probe Model 1 gave a perfusion value of $238 \pm 10$ a.u. and TLI of $0.85 \pm 0.02$ a.u. while probe Model 2 had a perfusion value of $227 \pm 11$ a.u. and TLI of $0.66 \pm 0.01$ a.u.

Figure 3a presents the perfusion signal during a skin temperature provocation using probe Model 1 and probe Model 2. The probes were placed one mm apart on the face of the right arms palm on top of Abductor pollicis brevis. Figure $3 \mathrm{~b}$ displays the corresponding perfusion trend curves using $10 \mathrm{~s}$ averages. Average perfusion, PI and HR for each $1 \mathrm{~min}$ period is presented below the trend curves. It is seen that the curves are following each other. The deviations in perfusion values are due to the tissue inhomogeneity [37].

\subsection{Evaluation during neurosurgery}

A summary of the tumor grade, used probe model and number of measurements sites for the-patients is found in Table 1. One of the patients was excluded (Patient 7) from the study due to technical difficulties as one optical probe did not receive enough light for the LDPM system (TLI <0.5) to produce a perfusion signal. In the remaining nine patients, recordings during surgery were fulfilled without technical failure and complications. At surgery one suspected brain tumor (Patient 2) was revealed as an abscess due to an infection and thus only measurements in white and grey matter were considered.

In Fig. 4 a-c an example of $20 \mathrm{~s}$ online recorded signals from grey matter, white matter and tumour tissue are displayed, originating from Patient 4. The mean values of perfusion, TLI, HR and PI over the $20 \mathrm{~s}$ recordings are presented in the notation-boxes. It is clearly seen that the TLI is higher in white matter compared to grey and that the reverse relation is found for the perfusion. In this specific case, the tumor presented an intermediate TLI with a clear pulsatile perfusion. The estimated HR from the perfusion signal corresponding well with the normal monitored heart rate in the operating theatre. 
The scatterplot including the average perfusion from all 76 measurement sites between perfusion and TLI for white, grey and tumor tissue is presented in Fig. 5. Recordings were made from different tissue sites classified by the surgeon as to originate from tumor $(n=28)$, white matter $(n=16)$ or grey matter $(n=22)$. Ten values were excluded as they were not clearly classified into a tissue type by the surgeon's visual inspection. The authors suggest a TLI threshold of 1.7 a.u. in order to separate between white and grey matter in non-tumorous tissue sites in this patient group.

Figure 6 show box plots of the cerebral perfusion and total backscatter in the three tissue types as sorted by the surgeon's visual inspection. The median perfusion was highest in tumor tissue (109 a.u.) followed by grey matter (96 a.u.) while the lowest perfusion was found in white matter (42 a.u.). Significant difference in median perfusion was found between grey and white matter $\left(\mathrm{p}<0.005, \mathrm{n}_{1}=22, \mathrm{n}_{2}=16\right)$. Likewise tumor and white tissue $\left(\mathrm{p}<0.001, \mathrm{n}_{1}=28, \mathrm{n}_{2}=16\right)$ presented a significant difference in terms of perfusion. No significant difference in blood flow was found between grey and tumor tissue. The median TLI was highest in white matter (2.59 a.u.) with less than half as much in tumor tissue (1.13 a.u.) and approximately one third of that in grey matter (0.86 a.u.) respectively. The TLI values of the tissue groups showed significant difference $\left(\mathrm{p}<0.05, \mathrm{n}_{1}=22\right.$, $\mathrm{n}_{2}=16, \mathrm{n}_{3}=28$ ) between all groups.

The patient specific perfusion, TLI and PI are presented as a set of boxplots in Fig. 7 ac. The results display the difference and data variability in perfusion, TLI and PI between patients. The PI showed a significant difference in medians between white matter and tumor tissue $\left(\mathrm{p}<0.05, \mathrm{n}_{1}=16\right.$, $\mathrm{n}_{2}=28$ ) but no difference between the other groups using Mann-Whitney tests. There was a good agreement between the HR estimated from the perfusion signal and the patient monitoring system in the operating theatre $(\mathrm{r}=0.80, \mathrm{p}<0.01, \mathrm{n}=18)$

\section{Discussion}

This study shows that the optical measurement system, with custom made software and a specialised fibre optical probe, is capable of monitoring of cerebral microcirculation during brain surgery. Due to the difficulty in distinguishing between brain tissue types with the naked eye, a quantitative way of differentiation based on the TLI value was suggested. Analysis of the recorded data at sites where the surgeon gave a clear tissue classification resulted in a threshold between cerebral grey and white matter. In addition, a significant difference in TLI between tumour and white matter was found. This in combination with preoperative CT or MRI may help to improve the intraoperative navigation and localization of tumor tissue for areas embedded in white matter and obstructed from clear view. According to other studies using PET it has been found that malignant tumours with higher grades has higher blood flow compared to the normal surrounding tissue over tumours with a lower grade $[34,8]$. 
Using the LDPM technique as guide during surgical interventions has thereby potential to give the surgeon instant information about the tumour type.

The LDPM signals and the derived parameters (perfusion, TLI, HR and PI) has the potential to become a useful complement to existing intraoperative navigational techniques as well as for monitoring in postoperative care by providing information regarding local microcirculation in the sampled brain tissue volume. During this evaluation study the cerebral microvascular blood flow was found to be significantly higher in grey matter and tumor tissue compared to white matter. This is in accordance to previous studies were also grey and white matter could be separated by using the TLI $[9,17,36,38]$. It must thus be pointed out that the TLI-threshold set in this study, may vary between individuals and also due to probe configuration. In this case the latter assumption can be neglected as the same fibre type and distance between fibres were used for the two probe models. In order to establish a more general TLI-threshold further investigations could include recordings from a larger patient group. However, for a feasibility study of the LDPM system during neurosurgery using a flexible fibre optic probe the number of samples are considered sufficient.

It is well known that LDPM signals can be affected by movement artefacts. This is especially important to consider when using a handheld probe that will be influenced by the hand movements of the surgeon. This problem was dealt with by releasing the probe after proper placement at a selected tissue site. During longer measurement periods the probe could be sutured into place to further reduce movement influences of the signal resulting in a stable and reliable blood flow recording. The design of the optical probe is highly important when measuring regional microcirculation. Much effort and knowledge gained from previous work [19,27] has been put into use in order to make the probe small, flexible enough to suit the application. The advantage of Model 2 over Model 1 is the exterior probe dimensions which were slightly reduced while still flexible with a rounded tip. The fibre distances were kept the same resulting in similar sampling volumes. Previous studies based on Monte Carlo simulations using similar optical probe tip designs have estimated the optical sampling depth to approximately $1 \mathrm{~mm}$ in brain tissue $[16,9,18]$. The probe design could also be compared with previous investigations where a subdural optode-strip was used on the brain surface [7,35]. As the LDPM system can be equipped with multiple detectors our configuration also allows for multipoint recordings, but with the added advantage of measurement possibilities inside the tissue.

For future application the aim is to use the LDPM-system for long term monitoring of CBF in the neurointensive care, displaying both HR and PI together with the perfusion and TLI. The heart rate estimation extracted from the LDPM signal was compared to the existing patient monitoring system in the operating theatre and showed good agreement which indicates that the software works as intended. Pulsatility index, commonly used in ultrasound and transcranial Doppler equipment, is 
proposed to reflect the level of vascular resistance in the circulatory system [32], however this is a question of debate in cerebral measurements. The PI-value will respond to drastic changes in the level of microvascular blood flow. This means that PI could be useful in detecting changes in the microcirculation during bedside monitoring which indicate a possible way of detecting reduced blood flow in the event of brain impairment. The software developed for neuromonitoring purposes used in this study could be used to quickly assess patient status when monitoring during longer periods. As changes over time often are relatively slow, trend curves can be built using averages over different time intervals e.g. from 10 to 60 seconds to keep track of and foresee dangerous levels of blood flow.

By combining the LDPM method with diffuse reflectance spectroscopy both blood flow and the oxygen saturation can be recorded as regional microcirculation parameters. This type of multimodal monitoring will give information about local oxygen delivery and utilization in the target area. Future work aim at measurements over longer periods (hours to a few days) in the neurointensive care unit where the probe is introduced during surgery for postoperative monitoring for example in the case of traumatic brain injury or subarachnoid haemorrhage. Additional combinations can involve pressure and microdialysis recordings. LDPM also has a potential to be used as a navigational supplement, due to tissue discrimination capabilities, in combination with other optical methods in neurosurgical applications, such as fluorescence guidance during brain tumor resection [12,30,28].

\section{Conclusion}

A LDPM system with custom designed probe and software has been adapted for measuring cerebral microcirculation. The system was evaluated on skin and intraoperative in brain tissue during neurosurgery. The study showed that the system could successfully be used as an intraoperative tool for monitoring local blood flow and several parameters linked to cerebral microcirculation (perfusion, TLI, HR and PI). Furthermore, the systems stability opens up for studies in the postoperative care of patients with e.g. traumatic brain injury or subarachnoid haemorrhage.

\section{Acknowledgement}

The authors would like to thank the clinical staff at the Neurosurgical Department of Linköping University Hospital for their help during the surgical measurements. The authors also acknowledge research engineer Mats Andersson and Per Sveider at the Department of Biomedical Engineering for skilful fabrication of the custom optical probes. This study was supported by the Swedish Research Council (Grant No. 6212-010-4216).

The authors declare that they have no conflict of interest. 


\section{References}

1. Arshi B, Mack WJ, Emanuel B (2013) Invasive and noninvasive multimodal bedside monitoring in subarachnoid hemorrhage: a review of techniques and available data. Neurology research international 2013:987934. doi:10.1155/2013/987934

2. Barazangi N, Hemphill JC, 3rd (2008) Advanced cerebral monitoring in neurocritical care. Neurology India 56 (4):405-414

3. Barone DG, Czosnyka M (2014) Brain Monitoring: Do We Need a Hole? An Update on Invasive and Noninvasive Brain Monitoring Modalities. TheScientificWorldJournal 2014:795762. doi:10.1155/2014/795762

4. Bellner J, Romner B, Reinstrup P, Kristiansson KA, Ryding E, Brandt L (2004) Transcranial Doppler sonography pulsatility index (PI) reflects intracranial pressure (ICP). Surgical neurology $62 \quad$ (1):45-51; discussion 51. doi:10.1016/j.surneu.2003.12.007

5. Czosnyka M, Pickard JD (2004) Monitoring and interpretation of intracranial pressure. Journal of neurology, neurosurgery, and psychiatry 75 (6):813-821

6. Dias C, Maia I, Cerejo A, Varsos G, Smielewski P, Paiva JA, Czosnyka M (2013) Pressures, Flow, and Brain Oxygenation During Plateau Waves of Intracranial Pressure. Neurocritical care. doi:10.1007/s12028-013-9918-y

7. Dreier JP, Major S, Manning A, Woitzik J, Drenckhahn C, Steinbrink J, Tolias C, OliveiraFerreira AI, Fabricius M, Hartings JA, Vajkoczy P, Lauritzen M, Dirnagl U, Bohner G, Strong AJ (2009) Cortical spreading ischaemia is a novel process involved in ischaemic damage in patients with aneurysmal subarachnoid haemorrhage. Brain : a journal of neurology 132 (Pt 7):1866-1881. doi:10.1093/brain/awp102

8. Ellika SK, Jain R, Patel SC, Scarpace L, Schultz LR, Rock JP, Mikkelsen T (2007) Role of perfusion CT in glioma grading and comparison with conventional MR imaging features. AJNR American journal of neuroradiology 28 (10):1981-1987. doi:10.3174/ajnr.A0688

9. Giller CA, Liu HL, Gurnani P, Victor S, Yasdani U, German DC (2003) Validation of a near-infrared probe for detection of thin intracranial white matter structures. J Neurosurg 98:1299-1306

10. Gosling RG, King DH (1974) Arterial assessment by Doppler-shift ultrasound. Proceedings of the Royal Society of Medicine 67 (6 Pt 1):447-449 
11. Haberl RL, Villringer A, Dirnagl U (1993) Applicability of laser-Doppler flowmetry for cerebral blood flow monitoring in neurological intensive care. Acta neurochirurgica Supplementum 59:64-68

12. Haj-Hosseini N, Richter J, Andersson-Engels S, Wårdell K (2010) Optical touch pointer for fluorescence guided glioblastoma resection using 5-aminolevulinic acid. Lasers Surg Med 42 (1):9-14

13. Hillman EM (2007) Optical brain imaging in vivo: techniques and applications from animal to man. J Biomed Opt 12 (5):051402

14. Hillman J, Sturnegk P, Yonas H, Heron J, Sandborg M, Gunnarsson T, Mellergard P (2005) Bedside monitoring of CBF with xenon-CT and a mobile scanner: a novel method in neurointensive care. $\mathrm{Br}$ J Neurosurg 19 (5):395-401. doi:10.1080/02688690500389898

15. Jacobs P (2006) Sterrad 100S, Sterilisation system. Johnson \& Johnson

16. Jakobsson A, Nilsson GE (1993) Prediction of sampling depth and photon pathlength in laser Doppler flowmetry. Med Biol Eng Comput 31 (3):301-307

17. Johansson JD, Blomstedt $\mathrm{P}$, Haj-Hosseini N, Bergenheim AT, Eriksson $\mathrm{O}$, Wårdell $\mathrm{K}$ (2009) Combined Diffuse Light Reflectance and Electrical Impedance Measurements as a Navigation Aid in Deep Brain Surgery. Stereotact Funct Neurosurg 87 (2):105113

18. Johansson JD, Fredriksson I, Wårdell K, Eriksson O (2009) Simulation of reflected light intensity changes during navigation and radio-frequency lesioning in the brain. $\mathrm{J}$ Biomed Opt 14 (4):044040

19. Karlsson MG, Casimir-Ahn H, Lönn U, Wårdell K (2003) Analysis and processing of laser Doppler perfusion monitoring signals recorded from the beating heart. Med Biol Eng Comput 41 (3):255-262

20. Keller E, Froehlich J, Muroi C, Sikorski C, Muser M (2011) Neuromonitoring in intensive care: a new brain tissue probe for combined monitoring of intracranial pressure (ICP) cerebral blood flow (CBF) and oxygenation. Acta neurochirurgica Supplement $110(\mathrm{Pt}$ 2):217-220. doi:10.1007/978-3-7091-0356-2_39

21. Louis DN, Ohgaki H, Wiestler OD, Cavenee WK, Burger PC, Jouvet A, Scheithauer BW, Kleihues P (2007) The 2007 WHO classification of tumours of the central nervous system. Acta neuropathologica 114 (2):97-109. doi:10.1007/s00401-007-0243-4 
22. Nasrallah I, Dubroff J (2013) An overview of PET neuroimaging. Seminars in nuclear medicine 43 (6):449-461. doi:10.1053/j.semnuclmed.2013.06.003

23. Nilsson GE, Salerud GE, Strömberg NOT, Wårdell K (2003) Laser Doppler perfusion monitoring and imaging. In Biomedical Photonics Handbook:Chapter 15, 11-24

24. Nilsson GE, Tenland T, Oberg PA (1980) Evaluation of a laser Doppler flowmeter for measurement of tissue blood flow. IEEE transactions on bio-medical engineering 27 (10):597-604. doi:10.1109/TBME.1980.326582

25. Parthasarathy AB, Weber EL, Richards LM, Fox DJ, Dunn AK (2010) Laser speckle contrast imaging of cerebral blood flow in humans during neurosurgery: a pilot clinical study. Journal of biomedical optics 15 (6):066030. doi:10.1117/1.3526368

26. Raabe A, Van De Ville D, Leutenegger M, Szelenyi A, Hattingen E, Gerlach R, Seifert V, Hauger C, Lopez A, Leitgeb R, Unser M, Martin-Williams EJ, Lasser T (2009) Laser Doppler imaging for intraoperative human brain mapping. NeuroImage 44 (4):12841289. doi:10.1016/j.neuroimage.2008.10.049

27. Rejmstad P, Akesson G, Hillman J, Wardell K (2012) A laser Doppler system for monitoring of intracerebral microcirculation. Conference proceedings : Annual International Conference of the IEEE Engineering in Medicine and Biology Society IEEE Engineering in Medicine and Biology Society Conference 2012:1988-1991. doi:10.1109/EMBC.2012.6346346

28. Richter JC, Haj-Hosseini N, Andersson-Engel S, Wardell K (2011) Fluorescence spectroscopy measurements in ultrasonic navigated resection of malignant brain tumors. Lasers Surg Med 43 (1):8-14. doi:10.1002/lsm.21022

29. Rostami E, Engquist H, Enblad P (2014) Imaging of cerebral blood flow in patients with severe traumatic brain injury in the neurointensive care. Frontiers in neurology 5:114. doi:10.3389/fneur.2014.00114

30. Stummer W, Pichlmeier U, Meinel T, Wiestler OD, Zanella F, Reulen HJ (2006) Fluorescence-guided surgery with 5-aminolevulinic acid for resection of malignant glioma: a randomised controlled multicentre phase III trial. The lancet oncology 7 (5):392-401. doi:10.1016/S1470-2045(06)70665-9

31. Su XW, Guan Y, Barnes M, Clark JB, Myers JL, Undar A (2011) Improved cerebral oxygen saturation and blood flow pulsatility with pulsatile perfusion during pediatric cardiopulmonary bypass. Pediatric research 70 (2):181-185. doi:10.1038/pr.2011.406 
32. Tarumi T, Ayaz Khan M, Liu J, Tseng BM, Parker R, Riley J, Tinajero C, Zhang R (2014) Cerebral hemodynamics in normal aging: central artery stiffness, wave reflection, and pressure pulsatility. Journal of cerebral blood flow and metabolism : official journal of the International Society of Cerebral Blood Flow and Metabolism. doi:10.1038/jcbfm.2014.44

33. Wardell K, Jakobsson A, Nilsson GE (1993) Laser Doppler perfusion imaging by dynamic light scattering. IEEE transactions on bio-medical engineering 40 (4):309316. doi:10.1109/10.222322

34. Warmuth C, Gunther M, Zimmer C (2003) Quantification of blood flow in brain tumors: comparison of arterial spin labeling and dynamic susceptibility-weighted contrastenhanced MR imaging. Radiology 228 (2):523-532. doi:10.1148/radiol.2282020409

35. Winkler MK, Chassidim Y, Lublinsky S, Revankar GS, Major S, Kang EJ, OliveiraFerreira AI, Woitzik J, Sandow N, Scheel M, Friedman A, Dreier JP (2012) Impaired neurovascular coupling to ictal epileptic activity and spreading depolarization in a patient with subarachnoid hemorrhage: possible link to blood-brain barrier dysfunction. Epilepsia 53 Suppl 6:22-30. doi:10.1111/j.1528-1167.2012.03699.x

36. Wårdell K, Blomstedt $\mathrm{P}$, Richter J, Antonsson J, Eriksson O, Zsigmond $\mathrm{P}$, Bergenheim AT, Hariz MI (2007) Intracerebral microvascular measurements during deep brain stimulation implantation using laser Doppler perfusion monitoring. Stereotact Funct Neurosurg 85 (6):279-286

37. Wårdell K, Braverman IM, Silverman DG, Nilsson GE (1994) Spatial heterogeneity in normal skin perfusion recorded with laser Doppler imaging and flowmetry. Microvasc Res 48 (1):26-38

38. Wårdell K, Zsigmond P, Richter J, Hemm S (2013) Relationship between laser Doppler signals and anatomy during deep brain stimulation electrode implantation toward the ventral intermediate nucleus and subthalamic nucleus. Neurosurgery 72 (2 Suppl Operative):ons127-140. doi:10.1227/NEU.0b013e31827e5821

39. You J, Du C, Volkow ND, Pan Y (2014) Optical coherence Doppler tomography for quantitative cerebral blood flow imaging. Biomedical optics express 5 (9):3217-3230. doi:10.1364/BOE.5.003217 
40. Zweifel C, Dias C, Smielewski P, Czosnyka M (2014) Continuous time-domain monitoring of cerebral autoregulation in neurocritical care. Medical engineering \& physics. doi:10.1016/j.medengphy.2014.03.002 


\section{Tables and Figures}

Table 1 Patient diagnosis, probe model used for intraoperative measurement and number of recorded tissue sites for each patient

\begin{tabular}{llll}
\hline Patient & $\begin{array}{l}\text { Tumor } \\
\text { grade }\end{array}$ & $\begin{array}{l}\text { Probe } \\
\text { model }\end{array}$ & $\begin{array}{l}\# \\
\text { Sites }\end{array}$ \\
\hline 1 & Low & 1 & 10 \\
2 & $\begin{array}{l}\text { Non } \\
\text { tumor }\end{array}$ & 1 & 13 \\
3 & High & 1 & 7 \\
4 & Low & 1 & 12 \\
5 & High & 1 & 5 \\
6 & Low & 1 & 0 \\
7 & Low & 1 & 10 \\
8 & High & 2 & 10 \\
9 & High & 2 & 4 \\
10 & High & & \\
\hline
\end{tabular}


a)

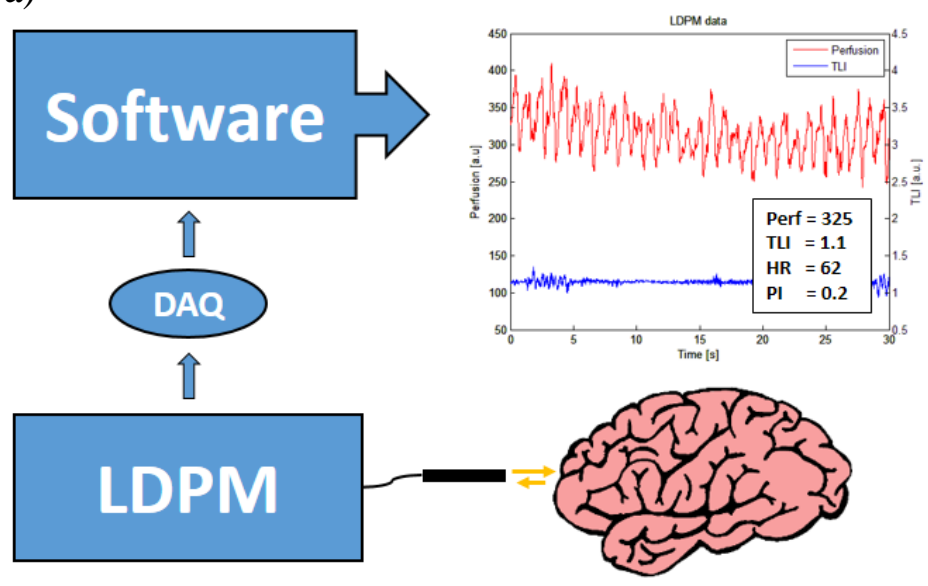

b)

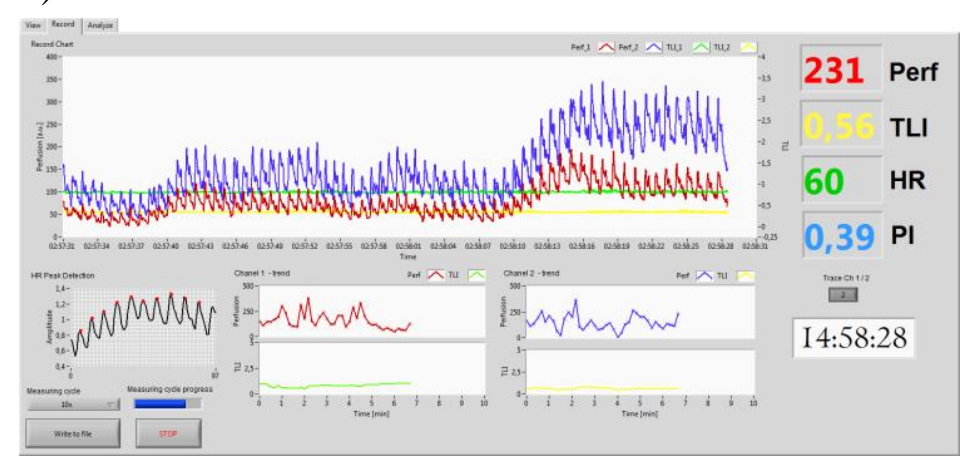

c)

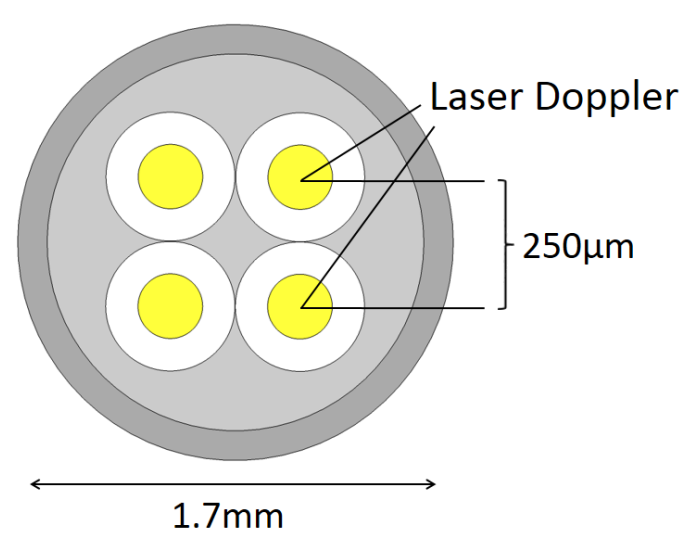

Fig. 1 a) Overview of the monitoring system, b) 
a)

$\bigcirc$ scrub

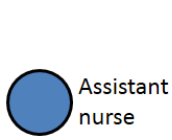

burgeon

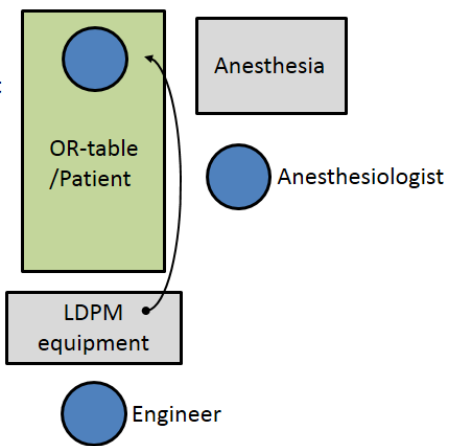

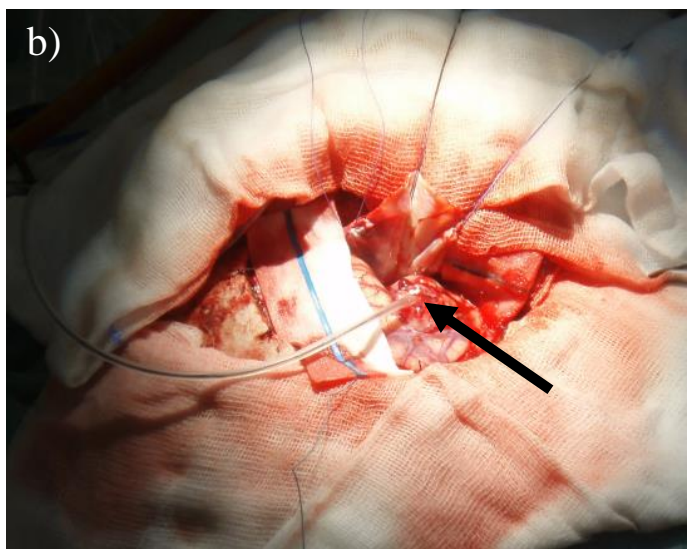

Fig. 2 Evaluation on brain tissue during tumor resection a) operating theatre setup and b) probe positioned at the surgical site

a)

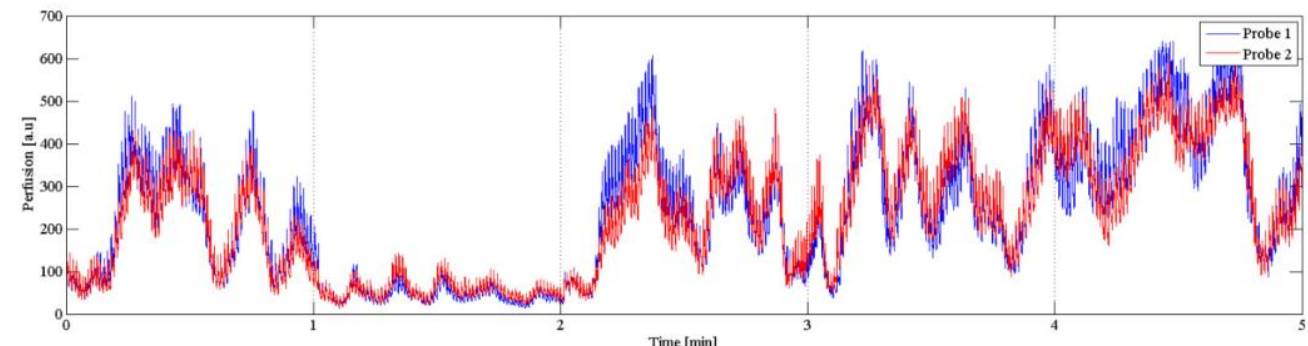

b)

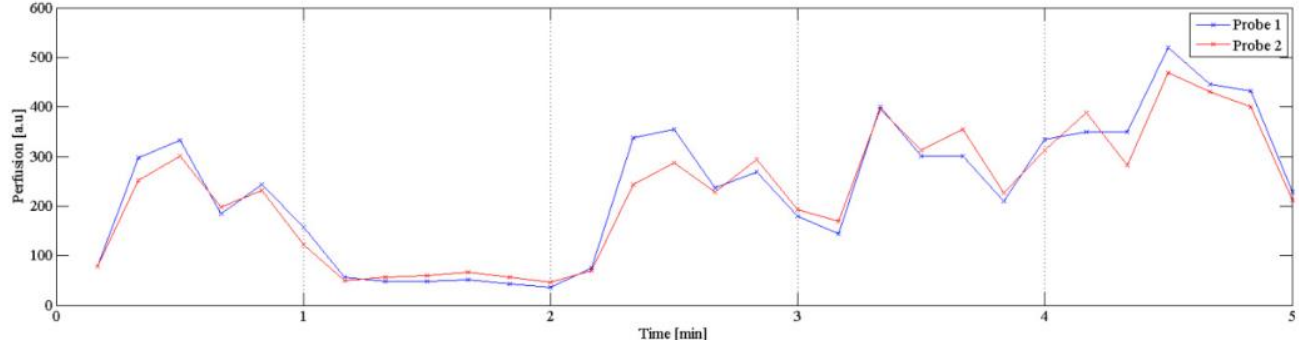

\begin{tabular}{l|l:l|l:l:l|} 
& Perf $=216$ & Perf $=47$ & Perf $=242$ & Perf $=282$ & Perf $=388$ \\
Probe 1 & PI $=0,28$ & PI $=0,44$ & PI $=0,36$ & PI $=0,59$ & PI $=0,44$ \\
& HR $=72$ & HR $=71$ & HR $=77$ & HR $=72$ & HR $=68$ \\
\hline \multirow{3}{*}{ Probe 2 } & Perf $=197$ & Perf $=56$ & Perf $=220$ & Perf $=295$ & Perf $=364$ \\
& PI $=0,23$ & PI $=0,39$ & PI $=0,40$ & PI $=0,50$ & PI $=0,48$ \\
& HR $=72$ & HR $=71$ & HR $=76$ & HR $=72$ & HR $=68$
\end{tabular}

Fig. 3 Evaluation on skin using probe 1 and 2, heat provocation (between min 1-2) showing the blood flow response a) perfusion signals and b) perfusion trends of $10 \mathrm{~s}$ averages divided by 1 min sections where the Perf, HR and PI are listed below 


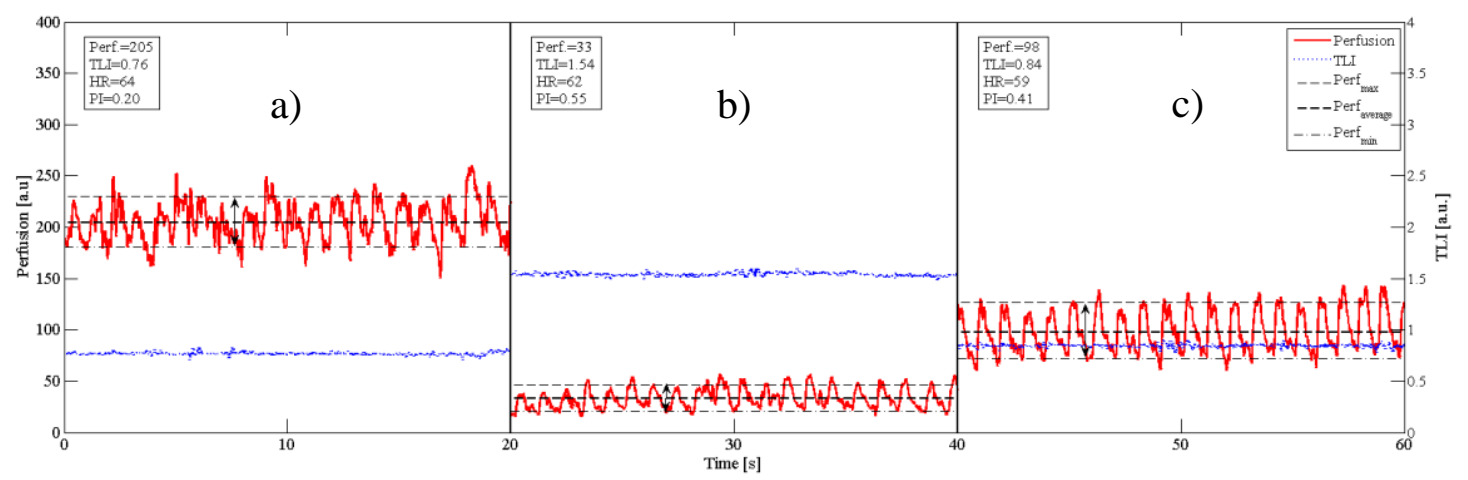

Fig. 4 LDPM signal with perfusion, TLI and derived parameters (notation box) from patient 4 in cerebral a) grey matter, b) white matter and c) tumor tissue, perfusion levels relevant to PI calculation are displayed using dashed horizontal lines

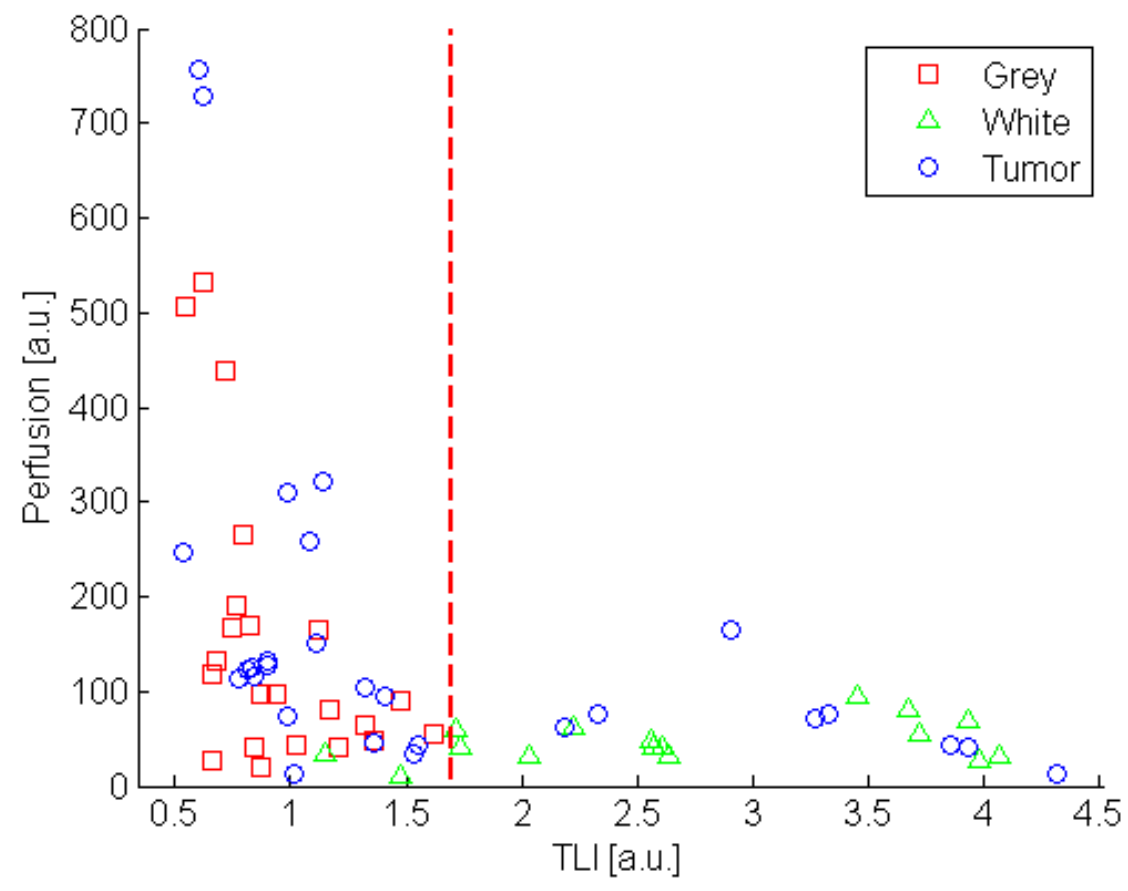

Fig. 5 Scatterplot showing LDPM data from measured tissue sites which are classified into grey, white or tumor tissue based on the surgeons visual inspection. The TLI threshold between grey and white matter is suggested to 1.7 a.u. 

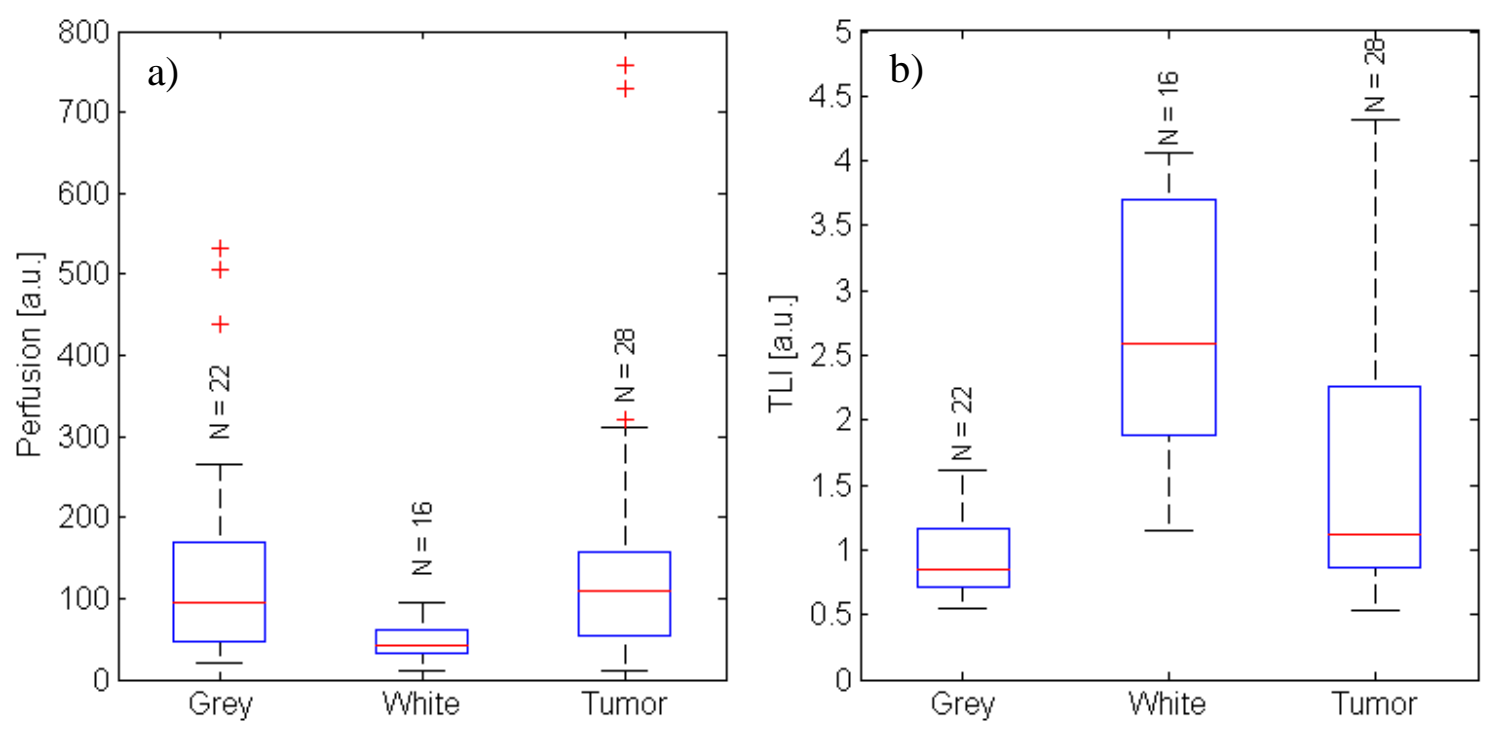

Fig. 6 Box plot with LDPM data showing medians of a) perfusion and b) TLI for the tissue types, grey matter $(n=22)$, white matter $(n=16)$ and tumor $(n=28)$ displaying $25 \%$ and $75 \%$ quartiles, whiskers and outliers 

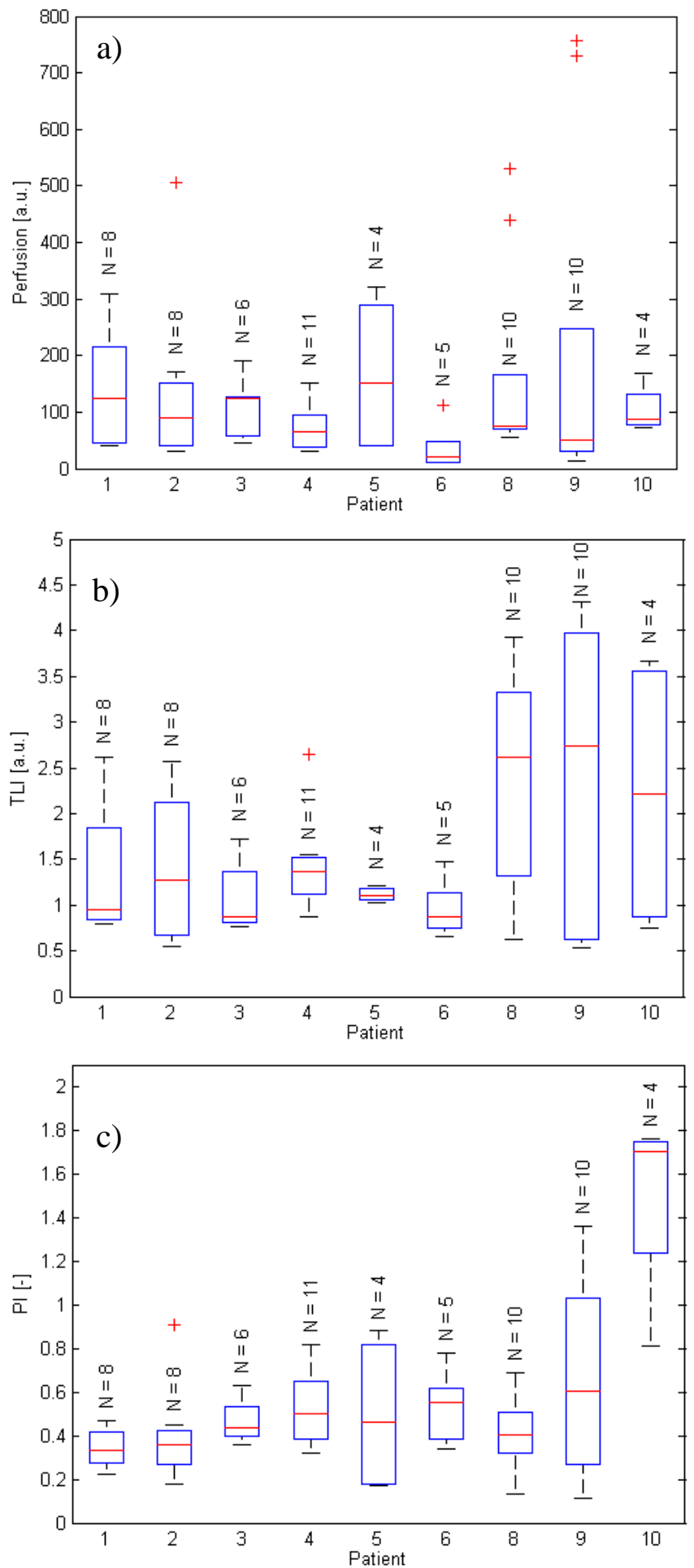

Fig. 7 Boxplot of patient specific a) perfusion, b) TLI and c) PI displaying $25 \%$ and $75 \%$ quartiles, whiskers and outliers 